\title{
Ferromagneto Toroidic Space Groups
}

\author{
M.Vijaya Laxmi ${ }^{1}$, S.Uma Devi
${ }^{2}$
PhD scholar, ${ }^{2}$ Professor, \\ Department of Engineering Mathematics, Andhra University, Visakhapatnam
}

\begin{abstract}
The full symmetry group of the crystal is called its Space group. The elements of space group are Combinations of the point group operations and translations. The 440 Ferroelectric space groups, viz the Heesch -shubnikov space groups, which are symmetry groups of Ferroelectric electric-dipole arrangements in crystals are already derived [4]. In this paper, we show that although Ferromagnetotoroidic point groups are 57, the number of Ferromagnetotoroidic spacegroups sum upto 728, and three Ferromagnetotoroidic spacegroups are tabulated by using Opechowski and Guccione symbols.
\end{abstract}

Keywords: space groups, Ferroelectric space groups, Ferromagnetotoroidic space groups, Magnetic space groups, point groups and grey groups

\section{INTRODUCTION}

D.B.Litvin has tabulated 440 Ferroelectric Space groups, via the Heesch - shubinkov (Magnetic) space groups. Opechowski and Guccione [4]derived 1191 types of Symbols of magnetic space groups [6] belov, et al, tabulated 1651 types of magnetic space groups, which are the direct product of a space group and the time inversion group. Here we list, Ferromagnetotoroidic space groups by using the Opechowski - guccione symbols. Aizu classified all possible ferroic phase transistions into 773 Species (Aizu, 1970) each species is given the Symbol $\mathrm{G}_{1}{ }^{1} \mathrm{~F} \mathrm{H}$ represents the transition between a paramagnetic phase point group symmetry $\mathrm{G}_{1}{ }^{1}$ and a lower symmetry phase of symmetry $\mathrm{H}$. The $\mathrm{F}$ denotes ferroic.

A fourth type of primary ferroic crystals, a ferrotoroidic crystal, has been recently observed (Van Aken etal, 2007) where the domains are distinguished by a toroidal moment (Gobatsevich et al 1983, Schmidt 2001, 2003a), is an extension of extend Aizu's species characterization and Schmidt's Classification of species into ensembles to include ferrotoroidic crystals by including the domain-state distinguish ability by a spontaneous toroidal moment.

So, in addition to the ferroelectric, ferromagnetic, and ferroelastic primay ferroic crystals where domain states differ respectively by spontaneous polarization, magnetization and strain, there is a observed fourth type of primary ferroic crystal (B.B.Van Aken etal ) where domain states differ in spontaneous toroidal moment (A.A.Gorbatsevich, H.Schmid etal, ) $\mathrm{T}_{(\mathrm{s})}$ is the spontaneous toroidal moment and Vector $\mathrm{S}$ with Components $\mathrm{s}_{\mathrm{i}} \sim$ $(\mathrm{E} \mathrm{X} \mathrm{H})_{i}$ is the source of the toroidal moment in the same way as electric and magnetic fields are source Vectors for Polarization and magnetization respectively. The physical property associated with each toroidic term is given in table 1 (D.B.Litvin 2008)

Table 1:

1. Spontaneous toroidal moment

2. Toroidic Susceptibility

3. Piezotoroidic Coefficient

4. Magneto toroidic Coefficient

5. Electrotoroidic Coefficient

6. Piezoelectric Coefficient

$\begin{array}{ll}\text { Ferrotoroidic } & \mathrm{av} \\ \text { Ferrobitoroidic } & {\left[\mathrm{V}^{2}\right]} \\ \text { Ferroelastotoroidic } & \mathrm{av}\left[\mathrm{V}^{2}\right] \\ \text { Ferromagentotoroidic } & \mathrm{eV}^{2} \\ \text { Ferroelectrotoroidic } & \mathrm{av}^{2} \\ \text { Ferroelastoelectric } & \mathrm{V}\left[\mathrm{v}^{2}\right]\end{array}$

Here first column given S.No Second column give physical property and $3^{\text {rd }} \& 4^{\text {th }}$ Columns give Corresponding Ferroic Type and John Symbol.

D.B.Litvin have tabulated 440 Ferroelectric Space groups, this work is extended to calculate Ferromagnetotoroidic Space group in Ferromagnetotoroidic $\left(\mathrm{eV}^{2}\right)$ property, "V" denotes polar Vector and "e" and "a" denotes zero-rank tensors that change sign under spatial inversion and time inversion respectively. Ferromagnetotoroidic point groups are 57, and they are given in Table - III. We then drive the 728 Ferromagnetotoroidic space groups (by using the opechowski \& guccione Symbols) and they are given in table IV. 


\section{CRYSTALLOGRAPHIC POINT GROUP}

Some Symmetry elements can exist together in the same crystal. There are 32 possible combinations altogether. A symmetric Survey of these combinations is called crystallographic point groups.

Grey Group: Let $\mathrm{G}$ be one of the 32 Crystallo-graphic point groups and $1^{1}$ consists of identity and time inversion operator $R_{2}$. The direct product of $\mathrm{G}$ and $1^{1}$, which is designated $\mathrm{G} 1{ }^{1}$ is known as grey group 32 point groups in which $R_{2}$ does not occur explicitly or in Combination with Symmetry operations are known as ordinary point groups.

Magnetic Point groups: The 58 groups in which R2 does not occur explicitly but occurs in combination with the symmetry operations are known as magnetic variants are (C.J.Bradly and Cracknel, 1972) of the 32 ordinary point groups. These 32 ordinary and 58 magnetic variants are known as magnetic point groups.

Space group: The full Symmetry group of a crystal is called its space group. The elements of spacegroup are Combinations of the point group operations and translations.

\section{FERROMAGNETOTOROIDIC SPACE GROUPS}

Let $\mathrm{F}$ denote a Crystallographic group type. The magnetic super family of Crystallographic groups of the type $\mathrm{F}$ (opechowski, 1986) consists of

a) Groups of type $\mathrm{F}$

b) Groups of type $\mathrm{F} 1{ }^{1}$, where $1^{1}$ denotes time inversion group consisting of the identity 1 and time inversion $1^{1}$

c) Groups of type F (D) $=\mathrm{D}+(\mathrm{F}-\mathrm{D}) 1^{1}$ where $\mathrm{D}$ is a Subgroup of index two of F.

Groups of this type will also be denoted by $\mathrm{M}^{2}$

The Third set of groups divided into two sub divisions.

i) Groups $\mathrm{M}_{\mathrm{T}}$, where $\mathrm{D}$ is an Equi-translation Subgroup of $\mathrm{F}$.

ii) Groups $M_{R}$ where $D$ is an equi-class subgroups of $F$.

Table II:

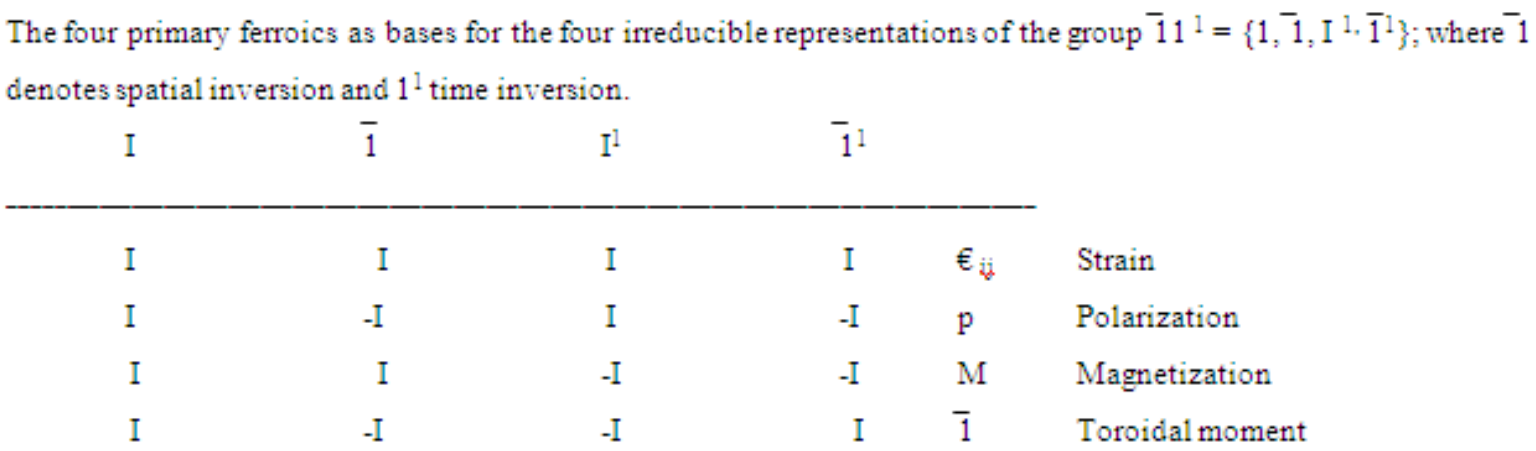

Further Let $\mathrm{P}, \mathrm{M}, \mathrm{T}$ and $€_{\mathrm{ij}}$ denote the four quantities polarization, Magnetization, Toroidal moment and Strain respectively (Aizu), Aizu have given all possible ferroic phase transitions into 773 species (Aizu, 1970). Each Species was characterized by three physical properties T, Spontaneous magnetization, polarization and strain. This is extended to the ferrotoroidic crystals which are given in table II. The characteristic transformation properties of a toroidal moment, along with that of other three primary Ferroics under elements of the group $\mathrm{T} \mathrm{I}^{\mathrm{I}}=\left\{\mathrm{I}, \mathrm{T}, \mathrm{I}^{\mathrm{I}}, \mathrm{T}^{\mathrm{I}}\right\}$ are given in table II . The toroidal moment is differentiating that it is reversed by both spatial and time inversion so, the toroidal moment tensor invariant under the group $\mathrm{H}$ of a species G1 ${ }^{1}$ FH.The 773 species of phase transistions $\mathrm{G} 1{ }^{1} \mathrm{FH}$ (Aizu 1970) respresent transitions between a paramagnetic phases of point group symmetry $\mathrm{G}_{1}{ }^{1}$ and a lower symmetry phase. The 773 species of phase transitions $\mathrm{G} 1^{1} \mathrm{FH}$ (Aizu, 1970) represent transitions between a paramagnetic phases of point group symmetry $\mathrm{G}_{1}{ }^{1}$ and a lower symmetry phase. Each species is characterized by four physical properties, ability to distinguish among the single domain states that arise due to the phase transition. These four physical properties are spontaneous toroidalmoment, spontaneous magnetization, spontaneous polarization, and spontaneous strain respectively. In the same manner the fourth types of primary and secondary Ferroic physical properties are given (3). So, here we determine the Ferromagnetotoroidoc point groups 57 and the corresponding Ferromagnetotoroidic space groups 645 (by sing opechowski and Guccione symbols), rest of the tables are available with the authors.

In table II, gives the character table of $11^{1}$ and classify the four quantities that appear in (Ferroic classification extended to Ferrotoroidic crystals) Maxwell's equations according to irreducible representations of the group $11^{1}$ 
If follows from character Table II and the vector properties of $\mathrm{P}, \mathrm{M}$ and $\mathrm{T}$ that the maximal symmetry group of a polarization vector $\mathrm{P}$ is $\infty \mathrm{m}_{1}^{\mathrm{I}}$ of a magnetization vector $\mathrm{M}$ is $\infty / \mathrm{mm}^{\mathrm{I}}$, and toroidalmanent vector $\infty / \mathrm{m}^{\mathrm{I}} \mathrm{m}$, this is Heesch - Shubnikov groups. Which Heesch - shubnikov groups have a point group that is a subgroup of $\infty \mathrm{m}_{1}{ }^{1}$. In a similar manner Heesch - Shubnikov groups have a point group that is a subgroup of $\infty / \mathrm{mm}^{\mathrm{I}}$, or $\infty / \mathrm{m}{ }^{\mathrm{I}} \mathrm{m}$. Here we list, Ferromagnetotoroidic space groups by using the opechowski \& Guccione Symbols, and they are given in table IV.

Table III: the fifty ferromagnetotoroidic point groups.

$\begin{array}{llll}1 & 1^{1} & & \\ 2 & 21^{1} & 2^{1} & \\ \mathrm{~m} & \mathrm{~m}_{1}{ }^{1} & \mathrm{~m}^{1} & \\ 222 & 2221^{1} & 2^{1} 2^{1} 2 & \\ \mathrm{~mm} 2 & \mathrm{~mm}^{1} 1^{1} & \mathrm{~m}^{1} \mathrm{~m} 2^{1} & \mathrm{~m}^{1} \mathrm{~m}^{1} 2 \\ 4 & 41^{1} & 4^{1} & \\ 4 & 41^{1} & 4^{1} & \\ 422 & 4221^{1} & 4^{1} 22^{1} & 42^{1} 2^{1} \\ 4 \mathrm{~mm} & 4 \mathrm{~mm}_{1}{ }^{1} & 4^{1} \mathrm{~m}^{1} \mathrm{~m} & 4 \mathrm{~m}^{1} \mathrm{~m}^{1} \\ 42 \mathrm{~m} & 42 \mathrm{~m}_{1}{ }^{1} & 4^{1} 2^{1} \mathrm{~m} & 42^{1} \mathrm{~m}^{1} \\ 3 & 31^{1} & & \\ 32 & 321^{1} & 32^{1} & \\ 3 \mathrm{~m} & 31^{1} & 3 \mathrm{~m}^{1} & \\ 6 & 61^{1} & 6^{1} & \\ 622 & 6221^{1} & 6^{1} 2^{1} 2 & 62^{1} 2^{1} \\ 6 \mathrm{~mm} & 6 \mathrm{~mm} 1^{1} & 6^{1} \mathrm{~m}^{1} \mathrm{~m} & 6 \mathrm{~m}^{1} \mathrm{~m}^{1} \\ 23 & 231^{1} & & \\ 432 & 4321^{1} & 4^{1} 32^{1} & \\ & & \end{array}$

Table IV: The 728 ferromagnetotoroidic point groups.

\begin{tabular}{|c|c|c|c|c|c|c|c|}
\hline Point group & 1 & $1^{1}$ & 2 & $2_{1}{ }^{1}$ & $2^{1}$ & $\mathbf{m}$ & $m_{1}{ }^{1}$ \\
\hline Space groups & $\mathrm{P} 1$ & $\mathrm{P}_{2 \mathrm{~s}} 1$ & $\begin{array}{l}\mathrm{P} 2 \\
\mathrm{P} 2_{1} \\
\mathrm{C} 2\end{array}$ & $\begin{array}{l}\mathrm{P}_{2 \mathrm{a}}{ }^{2} \\
\mathrm{P}_{2 \mathrm{~b}}{ }^{2} \\
\mathrm{P}_{\mathrm{c}}{ }^{2} \\
\mathrm{P}_{2 \mathrm{~b}} 2^{1} \\
\mathrm{P}_{2 \mathrm{a}} 2_{1} \\
\mathrm{C}_{2 \mathrm{c}} 2 \\
\mathrm{C}_{\mathrm{P}} 2 \\
\mathrm{C}_{\mathrm{P}} 2^{1} \\
\mathrm{P}_{2} 1^{1} \\
\mathrm{P}_{1} 1^{1} \\
\mathrm{C} 21^{1}\end{array}$ & $\begin{array}{l}\mathrm{P} 2^{1} \\
\mathrm{P} 2_{1}{ }^{1} \\
\mathrm{C} 2^{1}\end{array}$ & $\begin{array}{l}\mathrm{Pm} \\
\mathrm{Pc} \\
\mathrm{Cm} \\
\mathrm{Cc}\end{array}$ & $\begin{array}{l}\mathrm{P}_{2 \mathrm{a}} \mathrm{m} \\
\mathrm{P}_{2 b} \mathrm{~m} \\
\mathrm{P}_{\mathrm{c}} \mathrm{m} \\
\mathrm{P}_{2 \mathrm{c}} \mathrm{m}^{1} \\
\mathrm{P}_{2 \mathrm{a}} \mathrm{c} \\
\mathrm{P}_{2 \mathrm{~b}} \mathrm{c} \\
\mathrm{Pcc} \\
\mathrm{C}_{2 \mathrm{c}} \mathrm{m} \\
\mathrm{C}_{\mathrm{p}} \mathrm{m} \\
\mathrm{C}_{2 \mathrm{C}} \mathrm{m}^{1} \\
\mathrm{C}_{\mathrm{p}} \mathrm{m}^{1} \\
\mathrm{C}_{\mathrm{p}} \mathrm{c} \\
\mathrm{P} \mathrm{m}_{1}{ }^{1} \\
\mathrm{P} \mathrm{c}_{1}{ }^{1} \\
\mathrm{C} \mathrm{m}_{1}{ }^{1} \\
\mathrm{C} \mathrm{c}_{1}{ }^{1}\end{array}$ \\
\hline Point group & $\mathbf{m}^{1}$ & 222 & $2221^{1}$ & $2^{1} 2^{1} 2$ & $\mathbf{m m} 2$ & $\mathrm{~mm} 21^{1}$ & $\mathbf{m}^{1} \mathbf{m} 2^{1}$ \\
\hline Space groups & $\begin{array}{l}\mathrm{P} \mathrm{m^{1 }} \\
\mathrm{Pc}^{1} \\
\mathrm{Cm}^{1} \\
\mathrm{C} \mathrm{c}^{1}\end{array}$ & $\begin{array}{l}\text { P 222 } \\
\text { P 222 } \\
\text { P } 2_{1} 2_{1} 2 \\
\text { P } 2_{1} 2_{1} 2_{1} \\
\text { C } 222_{1} \\
\text { C } 222 \\
\text { F } 222 \\
\text { I } 222 \\
\text { I } 22_{1} 2_{1}\end{array}$ & $\begin{array}{l}\mathrm{P}_{2 \mathrm{a}} 222 \\
\mathrm{P}_{\mathrm{C}} 222 \\
\mathrm{P}_{\mathrm{F}} 222 \\
\mathrm{P}_{2 \mathrm{C}} 22^{1} 2^{1} \\
\mathrm{P}_{2 \mathrm{a}} 222_{1} \\
\mathrm{P}_{\mathrm{C}} 222_{1} \\
\mathrm{P}_{2 \mathrm{a}} 2^{1} 2^{1} 2_{1} \\
\mathrm{P}_{2 \mathrm{C}} 22_{1} 2 \\
\mathrm{P}_{2 \mathrm{C}} 22_{1} 2^{1}\end{array}$ & $\begin{array}{l}\mathrm{P} 2^{1} 2^{1} 2 \\
\mathrm{P} 2^{1} 2^{1} 2_{1} \\
\mathrm{P} 2{ }_{1}{ }^{1}{ }_{1}{ }^{1} \\
\mathrm{P} 2{ }_{1}{ }^{1}{ }_{1}{ }_{2} \\
\mathrm{C} 2^{1} 2^{1} 2_{1} \\
\mathrm{C} 2^{1} 2^{1}{ }^{1} \\
\mathrm{~F} 2^{1} 2^{1} 2 \\
\mathrm{I} 2^{1} 2^{1}{ }^{1} \\
\mathrm{I} 2_{1}{ }^{1}{ }_{1}{ }_{1}{ }^{1}{ }_{1}\end{array}$ & $\begin{array}{l}\text { Pmm2 } \\
\text { Pmc2 } \\
\text { Pcc2 } \\
\text { Pma2 } \\
\text { Pca2 }_{1} \\
\text { Pnc2 } \\
\text { Pmn2 } \\
\text { Pba2 } \\
\text { Pna2 }_{1} \\
\end{array}$ & $\begin{array}{l}\mathrm{P}_{2 \mathrm{c}} \mathrm{mm} 2 \\
\mathrm{P}_{2 \mathrm{a}} \mathrm{mm} 2 \\
\mathrm{P}_{\mathrm{c}} \mathrm{mm} 2 \\
\mathrm{P}_{\mathrm{A}} \mathrm{mm} 2 \\
\mathrm{P}_{\mathrm{F}} \mathrm{mm} 2 \\
\mathrm{P}_{2 \mathrm{c}} \mathrm{mm}^{1} 2^{1} \\
\mathrm{P}_{2 \mathrm{c}} \mathrm{m}^{1} \mathrm{~m}^{1} 2 \\
\mathrm{P}_{2 \mathrm{a}} \mathrm{m}^{1} \mathrm{~m}^{1} 2 \\
\mathrm{P}_{\mathrm{A}} \mathrm{m}^{1} \mathrm{~m}^{1} 2\end{array}$ & $\begin{array}{l}\mathrm{Pm}^{1} \mathrm{~m} 2^{1} \\
\mathrm{Pm}^{1}{ }^{1} 2_{1}{ }^{1} \\
\mathrm{Pm}^{1}{ }^{1} 2_{1}{ }^{1} \\
\mathrm{Pc}^{1} \mathrm{c}^{1}{ }^{1} \\
\mathrm{Pm}^{1}{ }^{1} 2^{1} \\
\mathrm{Pma}^{1} 2^{1} \\
\mathrm{Pc}^{1}{ }^{1} 2_{1}{ }^{1} \\
\mathrm{Pca}^{1} 2_{1}{ }^{1} \\
\mathrm{Pn}^{1} \mathrm{c}^{1}{ }^{1}\end{array}$ \\
\hline
\end{tabular}


Ferromagneto Toroidic Space Groups

\begin{tabular}{|c|c|c|c|c|c|c|c|}
\hline & & & $\begin{array}{l}\mathrm{C}_{\mathrm{P}} 222_{1} \\
\mathrm{C}_{\mathrm{P}} 2^{1} 2^{1}{ }^{1}{ }_{1} \\
\mathrm{C}_{2 \mathrm{C}} 222 \\
\mathrm{C}_{\mathrm{P}} 222 \\
\mathrm{C}_{1} 222 \\
\mathrm{C}_{2 \mathrm{C}} 22^{1} 2^{1} \\
\mathrm{C}_{\mathrm{P}} 2^{1} 2^{1} 2^{1} \\
\mathrm{C}_{\mathrm{P}} 22^{1} 2^{1} \\
\mathrm{C}_{1} 2^{1} 22^{1} \\
\mathrm{C}_{1} 2^{1} 22^{1} \\
\mathrm{~F}_{\mathrm{C}} 222 \\
\mathrm{~F}_{\mathrm{C}} 22^{1} 2^{1} \\
\mathrm{I}_{\mathrm{P}} 222 \\
\mathrm{I}_{\mathrm{P}} 22^{1} 2 \\
\mathrm{I}_{\mathrm{P}} 22_{1} 2_{1} \\
\mathrm{I}_{\mathrm{P}} 2_{1}{ }_{1}{ }_{1}{ }_{1}{ }_{1} \\
\mathrm{P} 2221^{1} \\
\mathrm{P} 222_{1} 1^{1} \\
\mathrm{P} 22_{1} 2_{1}{ }^{1} \\
\mathrm{P} 22_{1} 2_{1} 1^{1} \\
\mathrm{C} 222_{1} 1^{1} \\
\mathrm{C} 2221^{1} \\
\mathrm{~F} 2221^{1} \\
\text { I 222 } 1^{1} \\
\text { I } 22_{1} 2_{1} 1^{1}\end{array}$ & $\begin{array}{l}\text { P } 22^{1} 2_{1}{ }^{1} \\
\text { P } 2{ }_{1} 2_{1}{ }^{1}{ }^{1} \\
\text { C } 22^{1} 2_{1}{ }^{1} \\
\text { C } 22^{1} 2^{1}\end{array}$ & $\begin{array}{l}\text { Pnn2 } \\
\text { Cmm2 } \\
\text { Cmc2 } \\
\text { Ccc2 } \\
\text { Amm2 } \\
\text { Abm2 } \\
\text { Ama2 } \\
\text { Aba2 } \\
\text { Fdd2 } \\
\text { Imm2 } \\
\text { Iba2 } \\
\text { Ima2 }\end{array}$ & 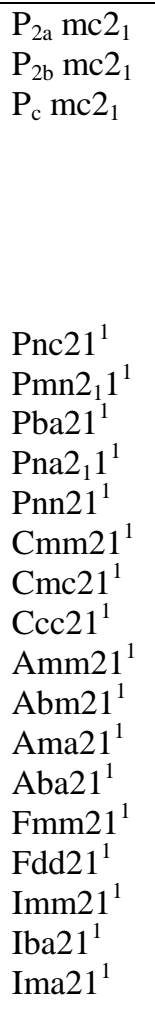 & 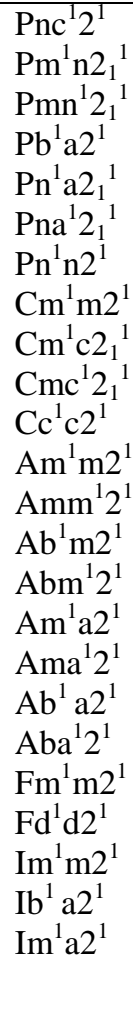 \\
\hline Point group & $\mathbf{m}^{1} \mathbf{m}^{1} \mathbf{2}$ & 4 & $41^{1}$ & $4^{1}$ & 4 & $41^{1}$ & $4^{1}$ \\
\hline Space groups & $\begin{array}{l}\mathrm{Pm}^{1} m^{1} 2 \\
\mathrm{Pm}^{1} c^{1} 2_{1} \\
\mathrm{Pc}^{1} c^{1} 2 \\
\mathrm{Pm}^{1} a^{1} 2 \\
\mathrm{Pc}^{1} a^{1} 2_{1} \\
\mathrm{Pn}^{1} c^{1}{ }^{1} \\
\mathrm{Pm}^{1} n^{1} 2_{1} \\
\mathrm{~Pb}^{1} a^{1} 2 \\
\mathrm{Pn}^{1} a^{1} 2_{1} \\
\mathrm{Pn}^{1} n^{1}{ }^{1} \\
\mathrm{Cm}^{1} m^{1} 2 \\
\mathrm{Cm}^{1} c^{1} 2_{1} \\
\mathrm{Cc}^{1} c^{1}{ }^{1} \\
\mathrm{Am}^{1} m^{1} 2 \\
\mathrm{Ab}^{1} m^{1} 2 \\
\mathrm{Am}^{1} a^{1} 2 \\
\mathrm{Ab}^{1} a^{1} 2 \\
\mathrm{Fm}^{1} m^{1} 2 \\
\mathrm{Fd}^{1} d^{1} 2 \\
\mathrm{Im}^{1} m^{1} 2 \\
\mathrm{Ib}^{1} \mathrm{a}^{1} 2 \\
\mathrm{Im}^{1} \mathrm{a}^{1} 2\end{array}$ & $\begin{array}{l}\mathrm{P}_{4} \\
\mathrm{P} 4_{1} \\
\mathrm{P} 4_{2} \\
\mathrm{P} 4_{3} \\
\mathrm{I} 4 \\
\mathrm{I}_{4}\end{array}$ & $\begin{array}{l}\mathrm{P}_{2 \mathrm{C}} 4 \\
\mathrm{P}_{\mathrm{P}} 4 \\
\mathrm{P}_{1} 4 \\
\mathrm{P}_{2 \mathrm{C}} 4^{1} \\
\mathrm{P}_{\mathrm{P}} 4_{1} \\
\mathrm{P}_{2 \mathrm{C}} 4_{2} \\
\mathrm{P}_{\mathrm{P}} 4_{2} \\
\mathrm{P}_{1} 4_{2} \\
\mathrm{P}_{2 \mathrm{C}} 4_{2}{ }^{1} \\
\mathrm{P}_{\mathrm{P}} 4_{3} \\
\mathrm{I}_{\mathrm{P}} 4 \\
\mathrm{I}_{\mathrm{P}} 4^{1} \\
\mathrm{I}_{\mathrm{P}} 4_{1} \\
\mathrm{I}_{\mathrm{P}} 4_{1}{ }^{1} \\
\mathrm{P} 41^{1} \\
\mathrm{P}_{1} 1^{1} \\
\mathrm{P}_{2} 1^{1} \\
\mathrm{P}_{3} 1^{1} \\
\mathrm{I}_{4} 1^{1} \\
\mathrm{I}_{1} 1_{1} 1^{1}\end{array}$ & $\begin{array}{l}\mathrm{P} 4{ }^{1}{ }^{1} \\
\mathrm{P}{ }_{1}{ }^{1} \\
\mathrm{P} 4_{2}{ }^{1} \\
\mathrm{P}{ }_{3}{ }^{1} \\
\mathrm{I} 4^{1} \\
\mathrm{I} 4_{1}{ }^{1}\end{array}$ & $\begin{array}{l}\text { P } 4 \\
\text { I } 4\end{array}$ & $\begin{array}{l}\mathrm{P}_{2 \mathrm{C}} 4 \\
\mathrm{P}_{\mathrm{P}} 4 \\
\mathrm{P}_{1} 4 \\
\mathrm{I}_{\mathrm{P}} 4 \\
\mathrm{P} 41^{1} \\
\mathrm{I}_{4} 1^{1}\end{array}$ & $\begin{array}{l}\mathrm{P} 4^{1} \\
\mathrm{I} 4^{1}\end{array}$ \\
\hline Point group & 422 & $4221^{1}$ & $4^{1} 22^{1}$ & $42^{1} 2^{1}$ & $4 \mathrm{~mm}$ & $4 \mathrm{~mm}_{1}{ }^{1}$ & $4^{1} \mathrm{~m}^{1} \mathrm{~m}$ \\
\hline Space groups & $\begin{array}{l}\mathrm{P} 422 \\
\mathrm{P} 42_{1} 2 \\
\mathrm{P} 4_{1} 22 \\
\mathrm{P} 4_{1}{ }_{1} 2 \\
\end{array}$ & $\begin{array}{l}\mathrm{P}_{2 \mathrm{C}} 422 \\
\mathrm{P}_{\mathrm{P}} 422 \\
\mathrm{P}_{1} 422 \\
\mathrm{P}_{2 \mathrm{C}}{ }^{1} 22^{1}\end{array}$ & $\begin{array}{l}\mathrm{P} 4^{1} 22^{1} \\
\mathrm{P} 4^{1}{ }_{2} 2^{1} \\
\mathrm{P} 4_{1}^{1} 22^{1} \\
\mathrm{P} 4_{1}^{1} 2_{1} 2^{1}\end{array}$ & $\begin{array}{l}\mathrm{P} 42^{1} 2^{1} \\
\mathrm{P} 42_{1}{ }^{1} 2^{1} \\
\mathrm{P} 4{ }_{1} 2^{1} 2^{1} \\
\mathrm{P} 4{ }_{1} 2_{1} 2^{1}\end{array}$ & $\begin{array}{l}\mathrm{P} 4 \mathrm{~mm} \\
\mathrm{P} 4 \mathrm{bm} \\
\mathrm{P} 4_{2} \mathrm{~cm} \\
\mathrm{P} 4_{2} \mathrm{~nm}\end{array}$ & $\begin{array}{l}\mathrm{P}_{2 c} 4 \mathrm{~mm} \\
\mathrm{P}_{\mathrm{p}} 4 \mathrm{~mm} \\
\mathrm{P}_{1} 4 \mathrm{~mm} \\
\mathrm{P}_{2 \mathrm{c}} 4^{1} \mathrm{~m}^{1} \mathrm{~m}\end{array}$ & $\begin{array}{l}\mathrm{P} 4{ }^{1} \mathrm{~m}^{1} \mathrm{~m} \\
\mathrm{P} 4^{1} \mathrm{~mm}^{1} \\
\mathrm{P} 4^{1} \mathrm{~b}^{1} \mathrm{~m} \\
\mathrm{P} 4^{1} \mathrm{bm}^{1}\end{array}$ \\
\hline
\end{tabular}


Ferromagneto Toroidic Space Groups

\begin{tabular}{|c|c|c|c|c|c|c|c|}
\hline & $\begin{array}{l}\mathrm{P} 4_{2} 22 \\
\mathrm{P} 4_{2} 2_{1} 2 \\
\mathrm{P} 4_{3} 22 \\
\mathrm{P}_{3}{ }_{2} 2 \\
\mathrm{I} 422 \\
\mathrm{I} 4_{1} 22\end{array}$ & 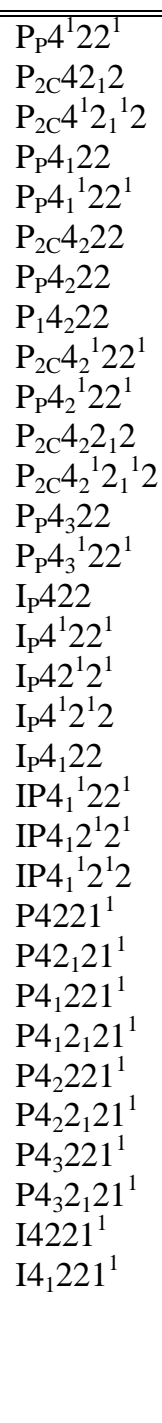 & $\begin{array}{l}\mathrm{P} 4_{2}{ }^{1} 22^{1} \\
\mathrm{P} 4_{2}{ }^{1} 2_{1} 2^{1} \\
\mathrm{P} 4_{3}{ }^{1} 22^{1} \\
\mathrm{P} 4_{3}{ }^{1} 22^{1} \\
\mathrm{I} 4^{1} 22^{1} \\
\mathrm{I} 4_{1}{ }^{1} 22^{1}\end{array}$ & $\begin{array}{l}\mathrm{P} 4_{2} 2^{1} 2^{1} \\
\mathrm{P} 4_{2} 2_{1}{ }^{1} 2^{1} \\
\mathrm{P} 4_{3} 2^{1} 2^{1} \\
\mathrm{P}_{4} 2_{1}{ }^{1} 2^{1} \\
\mathrm{I} 42^{1} 2^{1} \\
\mathrm{I} 4_{1} 2^{1} 2^{1}\end{array}$ & $\begin{array}{l}\mathrm{P} 4 \mathrm{cc} \\
\mathrm{P} 4 \mathrm{nc} \\
\mathrm{P} 4_{2} \mathrm{mc} \\
\mathrm{P} 4_{2} \mathrm{bc} \\
\mathrm{I} 4 \mathrm{~mm} \\
\mathrm{I} 4 \mathrm{~cm} \\
\mathrm{I}_{1} \mathrm{md} \\
\mathrm{I} 4_{1} \mathrm{~cd}\end{array}$ & 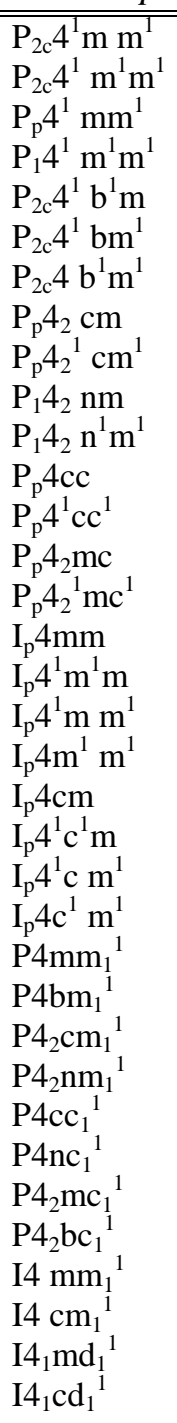 & 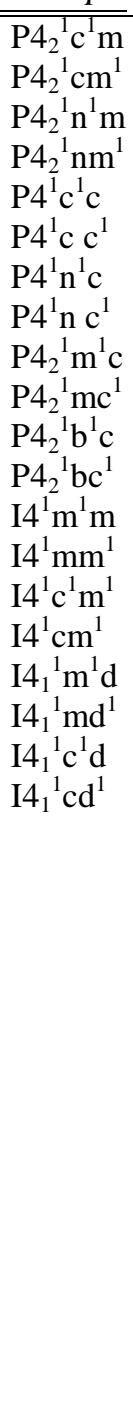 \\
\hline Point group & $4 m^{1} m^{1}$ & $42 \mathrm{~m}$ & $42 m_{1}^{1}$ & $4^{1} 2^{1} \mathrm{~m}$ & $42^{1} \mathrm{~m}^{1}$ & 3 & $31^{1}$ \\
\hline Space groups & $\begin{array}{l}\mathrm{P} 4 m^{1} m^{1} \\
\mathrm{P} 4 b^{1} m^{1} \\
\mathrm{P} 4_{2} c^{1} m^{1} \\
\mathrm{P} 4_{2} n^{1} m^{1} \\
\mathrm{P} 4 c^{1} c^{1} \\
\mathrm{P} 4 n^{1} c^{1} \\
\mathrm{P} 4_{2} \mathrm{~m}^{1} c^{1} \\
\mathrm{P} 4_{2} b^{1} c^{1} \\
\mathrm{I} \mathrm{m}^{1} \mathrm{~m}^{1} \\
\mathrm{I} \mathrm{c}^{1} \mathrm{~m}^{1} \\
\mathrm{I} 4_{1} \mathrm{~m}^{1} \mathrm{~d}^{1} \\
\mathrm{I} 4_{1} \mathrm{c}^{1} d^{1}\end{array}$ & $\begin{array}{l}\mathrm{P} 42 \mathrm{~m} \\
\mathrm{P} 42 \mathrm{c} \\
\mathrm{P} 42{ }_{1} \mathrm{~m} \\
\mathrm{P} 42{ }_{1} \mathrm{c} \\
\mathrm{P} 4 \mathrm{~m} 2 \\
\mathrm{P} 4 \mathrm{c} 2 \\
\mathrm{P} 4 \mathrm{~b} 2 \\
\mathrm{P} 4 \mathrm{n} 2 \\
\mathrm{I} 4 \mathrm{~m} 2 \\
\mathrm{I} 4 \mathrm{c} 2 \\
\mathrm{I} 42 \mathrm{~m}\end{array}$ & $\begin{array}{l}\mathrm{P}_{2 \mathrm{c}} 42 \mathrm{~m} \\
\mathrm{P}_{\mathrm{p}} 42 \mathrm{~m} \\
\mathrm{P}_{1} 42 \mathrm{~m} \\
\mathrm{P}_{2 \mathrm{c}} 2^{1} \mathrm{~m}^{1} \\
\mathrm{P}_{\mathrm{p}} 42 \mathrm{~m}^{1} \\
\mathrm{P}_{1} 42 \mathrm{~m}^{1} \\
\mathrm{P}_{\mathrm{p}} 42 \mathrm{c} \\
\mathrm{P}_{\mathrm{p}} 4{ }^{1} 2 \mathrm{c}^{1} \\
\mathrm{P}_{2 \mathrm{c}} 42_{1} \mathrm{~m} \\
\mathrm{P}_{2 \mathrm{c}} 4^{1} 2 \mathrm{~m}^{1} \\
\mathrm{P}_{2 \mathrm{c}} 4 \mathrm{~m} 2 \\
\mathrm{P}_{\mathrm{p}} 4 \mathrm{~m} 2 \\
\mathrm{P}_{1} 4 \mathrm{~m} 2\end{array}$ & $\begin{array}{l}\mathrm{P} 4^{1} 2^{1} \mathrm{~m} \\
\mathrm{P} 4^{1} 2^{1} \mathrm{c} \\
\mathrm{P} 4^{1} 2_{1}{ }^{1} \mathrm{~m} \\
\mathrm{P} 4^{1} 2_{1}{ }^{1} \mathrm{c} \\
\mathrm{P} 4^{1} \mathrm{~m} 2^{1} \\
\mathrm{P} 4^{1} \mathrm{c} 2^{1} \\
\mathrm{P} 4^{1} \mathrm{~b} 2^{1} \\
\mathrm{P} 4^{1} 2^{1} \\
\mathrm{I} 4^{1} \mathrm{~m}^{1} \\
\mathrm{I} 4^{1} \mathrm{c} 2^{1} \\
\mathrm{I} 4^{1} 2^{1} \mathrm{~m} \\
\mathrm{I} 4^{1} 2^{1} \mathrm{~d}\end{array}$ & $\begin{array}{l}\mathrm{P} 42^{1} \mathrm{~m}^{1} \\
\mathrm{P} 42^{1} \mathrm{c}^{1} \\
\mathrm{P} 42^{1}{ }_{1}^{1} \mathrm{~m}^{1} \\
\mathrm{P} 42^{1}{ }_{1} \mathrm{c}^{1} \\
\mathrm{P} 4 \mathrm{~m}^{1} 2^{1} \\
\mathrm{P} 4 \mathrm{c}^{1} 2^{1} \\
\mathrm{P} 4 \mathrm{~b}^{1} 2^{1} \\
\mathrm{P} 4 \mathrm{n}^{1} 2^{1} \\
\mathrm{I} 4 \mathrm{~m}^{1} 2^{1} \\
\mathrm{I} 4 \mathrm{c}^{1} 2^{1} \\
\mathrm{I} 42^{1} \mathrm{~m}^{1} \\
\mathrm{I} 42^{1} \mathrm{~d}^{1}\end{array}$ & $\begin{array}{l}\mathrm{P} 3 \\
\mathrm{P} 3_{1} \\
\mathrm{P} 3_{2} \\
\mathrm{R} 3\end{array}$ & $\begin{array}{l}\mathrm{P}_{2 \mathrm{C}} 3 \\
\mathrm{P}_{2 \mathrm{C}} 3_{2} \\
\mathrm{P}_{2 \mathrm{C}} 3_{3} \\
\mathrm{P}_{2 \mathrm{C}} 3_{1} \\
\mathrm{R}_{\mathrm{R}} 3 \\
\mathrm{P} 31^{1} \\
\mathrm{P} 3_{1} 1^{1} \\
\mathrm{P} 3_{2} 1^{1} \\
\mathrm{R} 31^{1}\end{array}$ \\
\hline
\end{tabular}




\begin{tabular}{|c|c|c|c|c|c|c|c|}
\hline & & $\mathrm{I} 42 \mathrm{~d}$ & $\begin{array}{l}\mathrm{P}_{2 c} 4^{1} \mathrm{~m}^{1} 2 \\
\mathrm{P}_{\mathrm{p}} 4^{1} \mathrm{~m}^{1} \\
\mathrm{P}_{\mathrm{p}} 4 \mathrm{c} 2 \\
\mathrm{P}_{\mathrm{p}} 4^{1} \mathrm{c} 2^{1} \\
\mathrm{P}_{2 \mathrm{c}} 4 \mathrm{~b} 2 \\
\mathrm{P}_{2 \mathrm{c}} 4 \mathrm{~b}^{1} 2 \\
\mathrm{P}_{1} 4 \mathrm{n} 2 \\
\mathrm{I}_{\mathrm{p}} 4 \mathrm{~m} 2 \\
\mathrm{I}_{\mathrm{p}} 4^{1} \mathrm{~m}^{1} 2 \\
\mathrm{I}_{\mathrm{p}} 4 \mathrm{c} 2 \\
\mathrm{I}_{\mathrm{p}} 4 \mathrm{c}^{1} 2^{1} \\
\mathrm{I}_{\mathrm{p}} 42 \mathrm{~m} \\
\mathrm{I}_{\mathrm{p}} 4^{1} 2^{1} \mathrm{~m} \\
\mathrm{I}_{\mathrm{p}} 4^{1} 2 \mathrm{~m}^{1} \\
\mathrm{I}_{\mathrm{p}} 42^{1} \mathrm{~m}^{1}\end{array}$ & & & & \\
\hline Point group & 32 & $321^{1}$ & $32^{1}$ & $3 m$ & $3 m 1^{1}$ & $3 m^{1}$ & \\
\hline Space groups & $\begin{array}{l}\mathrm{P} 312 \\
\mathrm{P} 321 \\
\mathrm{P} 3_{1} 12 \\
\mathrm{P} 3_{1} 21 \\
\mathrm{P} 3_{2} 12 \\
\mathrm{P} 3_{2} 21 \\
\mathrm{R} 32\end{array}$ & $\begin{array}{l}\mathrm{P}_{2 \mathrm{C}} 312 \\
\mathrm{P}_{2 \mathrm{C}} 321 \\
\mathrm{P}_{2 \mathrm{C}} 3_{2} 12 \\
\mathrm{P}_{2 \mathrm{C}} 3_{2} 21 \\
\mathrm{P}_{2 \mathrm{C}} 3_{1} 12 \\
\mathrm{P}_{2 \mathrm{C}} 3_{1} 21 \\
\mathrm{R}_{\mathrm{R}} 32 \\
\mathrm{P} 3121^{1} \\
\mathrm{P} 3211^{1} \\
\mathrm{P} 3_{1} 121^{1} \\
\mathrm{P}_{2} 121^{1} \\
\mathrm{P} 3_{2} 211^{1} \\
\mathrm{R} 321^{1}\end{array}$ & $\begin{array}{l}\mathrm{P} 312^{1} \\
\mathrm{P} 32^{1} 1 \\
\mathrm{P} 3_{1} 12^{1} \\
\mathrm{P} 3_{1} 2^{1} 1 \\
\mathrm{P} 3_{2} 12^{1} \\
\mathrm{P}{ }_{2} 2^{1} 1 \\
\mathrm{R} 32^{1}\end{array}$ & $\begin{array}{l}\text { P3m1 } \\
\text { P31m } \\
\text { P3c1 } \\
\text { P31c } \\
\text { R3m } \\
\text { R3c }\end{array}$ & $\begin{array}{l}\mathrm{P}_{2 \mathrm{C}} 3 \mathrm{~m} \\
\mathrm{P}_{2 \mathrm{C}} 3 \mathrm{~m}^{1}{ }_{1} \\
\mathrm{P}_{2 \mathrm{C}} 31 \mathrm{~m} \\
\mathrm{P}_{2 \mathrm{C}} 31 \mathrm{~m}^{1} \\
\mathrm{R}_{\mathrm{R}} 3 \mathrm{~m} \\
\mathrm{R}_{\mathrm{R}} 3 \mathrm{~m}^{1} \\
\mathrm{P} 3 \mathrm{~m} 1^{1} \\
\mathrm{P} 31 \mathrm{~m}_{1}{ }^{1} \\
\mathrm{P} 3 \mathrm{c} 11^{1} \\
\mathrm{R} 31 \mathrm{c} 1^{1} \\
\mathrm{R} 3 \mathrm{~m} 1^{1}\end{array}$ & $\begin{array}{l}\text { P3m } 11 \\
\text { P31 }{ }^{1} \\
\text { P3c } 1 \\
\text { P31 } 1 \\
\text { R3 }{ }^{1}{ }^{1} \\
\text { R3c }\end{array}$ & \\
\hline Point group & 6 & $61^{1}$ & $6^{1}$ & 622 & $6221^{1}$ & $6^{1} 2^{12}$ & $62^{1} 2^{1}$ \\
\hline Space groups & $\begin{array}{l}\mathrm{P} 6 \\
\mathrm{P} 6_{1} \\
\mathrm{P}_{5} \\
\mathrm{P} 6_{2} \\
\mathrm{P}_{4} \\
\mathrm{P} 6_{3}\end{array}$ & $\begin{array}{l}\mathrm{P}_{2 \mathrm{C}} 6 \\
\mathrm{P}_{2 \mathrm{C}} 6^{1} \\
\mathrm{P}_{2 \mathrm{C}} 6_{2} \\
\mathrm{P}_{2 \mathrm{C}} 6_{2}{ }^{1} \\
\mathrm{P}_{2 \mathrm{C}} 6_{4} \\
\mathrm{P}_{2 \mathrm{C}} 6^{1}{ }_{4} \\
\mathrm{P} 61^{1} \\
\mathrm{P}_{1} 1^{1} \\
\mathrm{P}_{5} 1^{1} \\
\mathrm{P}_{2} 1^{1} \\
\mathrm{P} 6_{4} 1^{1}\end{array}$ & $\begin{array}{l}\mathrm{P} 6^{1}{ }^{1} \\
\mathrm{P} 6_{1}{ }^{1} \\
\mathrm{P} 6_{5}{ }^{1} \\
\mathrm{P} 6_{2}{ }^{1} \\
\mathrm{P} 6_{4}{ }^{1} \\
\mathrm{P} 6_{3}{ }^{1}\end{array}$ & $\begin{array}{l}\mathrm{P} 622 \\
\mathrm{P}_{1} 22 \\
\mathrm{P}_{5} 22 \\
\mathrm{P}_{2} 22 \\
\mathrm{P}_{4} 22 \\
\mathrm{P} 6_{3} 22\end{array}$ & $\begin{array}{l}\mathrm{P}_{2 C} 622 \\
\mathrm{P}_{2 C} 6^{1} 22^{1} \\
\mathrm{P}_{2 C} 6_{2} 22 \\
\mathrm{P}_{2 C} 6_{2}{ }^{1} 22^{1} \\
\mathrm{P}_{2 C} 6_{4} 22 \\
\mathrm{P}_{2 C} 6_{4} 2^{1} 2 \\
\mathrm{P}_{2} 21^{1} \\
\mathrm{P}_{1} 221^{1} \\
\mathrm{P}_{5} 221^{1} \\
\mathrm{P}_{2} 221^{1} \\
\mathrm{P}_{4} 221^{1}\end{array}$ & $\begin{array}{l}\mathrm{P}^{1} 2^{1} 2 \\
\mathrm{P}_{1}{ }_{1}^{1} 2^{1} 2 \\
\mathrm{P}_{5} 2^{1} 2^{1} \\
\mathrm{P}_{2}{ }^{1} 2^{1} \\
\mathrm{P}_{4}{ }^{1} 2^{1} 2 \\
\mathrm{P}_{3}{ }^{1} 2^{1} 2\end{array}$ & $\begin{array}{l}\mathrm{P} 62^{1} 2^{1} \\
\mathrm{P}_{1} 2^{1} 2^{1} \\
\mathrm{P} 6_{5} 2^{1} 2^{1} \\
\mathrm{P}_{2} 2^{1} 2^{1} \\
\mathrm{P} 6_{4} 2^{1} 2^{1} \\
\mathrm{P} 6_{3} 2^{1} 2^{1}\end{array}$ \\
\hline
\end{tabular}


Ferromagneto Toroidic Space Groups

\begin{tabular}{|c|c|c|c|c|c|c|c|}
\hline & & $\mathrm{P}_{3}{ }_{1}{ }^{\mathrm{I}}$ & & & $\mathrm{P}_{3} 221^{\mathrm{I}}$ & & \\
\hline Point group & $6 \mathrm{~mm}$ & $6 \mathrm{~mm} 1^{1}$ & $6^{1} m^{1} m$ & $6 m^{1} m^{1}$ & 23 & $231^{1}$ & 432 \\
\hline Space groups & $\begin{array}{l}\mathrm{P} 6 \mathrm{~mm} \\
\mathrm{P} 6 \mathrm{cc} \\
\mathrm{P}_{3} \mathrm{~cm} \\
\mathrm{P} 6_{3} \mathrm{mc}\end{array}$ & $\begin{array}{l}\mathrm{P}_{2 \mathrm{C}} 6 \mathrm{~mm} \\
\mathrm{P}_{2 \mathrm{C}} 6^{1} \mathrm{~m}^{1} \mathrm{~m} \\
\mathrm{P}_{2 \mathrm{C}} 6^{1} \mathrm{~m} \mathrm{~m}^{1} \\
\mathrm{P}_{2 \mathrm{C}} 6 \mathrm{~m}^{1} \mathrm{~m}^{1} \\
{\mathrm{P} 6 \mathrm{~mm}_{1}{ }^{1}}{\mathrm{P} 6 \mathrm{cc}_{1}{ }^{1}} \mathrm{P}_{3} \mathrm{~cm}_{1}{ }^{1} \\
\mathrm{P}_{3} \mathrm{mc}_{1}{ }^{1}\end{array}$ & $\begin{array}{l}\mathrm{P} 6^{1} \mathrm{~m}^{\mathrm{I}} \mathrm{m} \\
\mathrm{P} 6^{1} \mathrm{~m} \mathrm{~m}^{1} \\
\mathrm{P}^{1}{ }^{1} \mathrm{c}^{1} \mathrm{c} \\
\mathrm{P}^{1}{ }^{1} \mathrm{cc}^{1} \\
\mathrm{P}_{3}{ }^{1} \mathrm{c}^{1} \mathrm{~m} \\
\mathrm{P} 6_{3}{ }^{1} \mathrm{~cm}^{1} \\
\mathrm{P} 6_{3}{ }^{1} \mathrm{~m}^{1} \mathrm{c} \\
\mathrm{P} 6_{3}{ }^{1} \mathrm{mc}^{1}\end{array}$ & $\begin{array}{l}\mathrm{P} 6 \mathrm{~m}^{1} \mathrm{~m}^{1} \\
\mathrm{P}^{1} \mathrm{c}^{1} \mathrm{c}^{1} \\
\mathrm{P6}_{3} \mathrm{c}^{1} \mathrm{~m}^{1} \\
\mathrm{P}_{3} \mathrm{~m}^{1} \mathrm{c}^{1}\end{array}$ & $\begin{array}{l}\mathrm{P} 23 \\
\mathrm{~F} 23 \\
\mathrm{I} 23 \\
\mathrm{P} 2{ }_{1} 3 \\
\mathrm{I}_{1} 3\end{array}$ & $\begin{array}{l}\mathrm{P}_{\mathrm{F}} 23 \\
\mathrm{I}_{\mathrm{P}} 23 \\
\mathrm{I}_{\mathrm{P}} 2_{1} 3 \\
\mathrm{P} 231^{1} \\
\mathrm{~F} 231^{1} \\
\mathrm{I} 231^{1} \\
\mathrm{P} 2_{1} 31^{1} \\
\mathrm{I}{ }_{1} 31^{1}\end{array}$ & $\begin{array}{l}\mathrm{P} 432 \\
\mathrm{P}_{2} 32 \\
\mathrm{~F} 432 \\
\mathrm{~F} 4_{1} 32 \\
\mathrm{I} 432 \\
\mathrm{P} 4_{3} 32 \\
\mathrm{P}_{1} 32 \\
\mathrm{I}_{1} 32\end{array}$ \\
\hline Point group & $4321^{1}$ & $4^{1} 32^{1}$ & & & & & \\
\hline Space groups & $\begin{array}{l}\mathrm{P}_{\mathrm{F}} 432 \\
\mathrm{P}_{\mathrm{F}} 4_{2} 32 \\
\mathrm{I}_{\mathrm{P}} 432 \\
\mathrm{I}_{\mathrm{P}} 4{ }^{1} 32^{1} \\
\mathrm{I}_{\mathrm{P}} 4_{1} 32 \\
\mathrm{I}_{\mathrm{P}} 4_{1}{ }_{1} 32 \\
\mathrm{P} 4321^{1} \\
\mathrm{P}_{2} 4_{2} 321^{1} \\
\mathrm{~F} 4321^{1} \\
\mathrm{I} 4321^{1} \\
\mathrm{P}_{3} 321^{1} \\
\mathrm{I}_{3} 321^{1}\end{array}$ & $\begin{array}{l}\mathrm{P} 4^{1} 32^{1} \\
\mathrm{P} 44_{2}^{1} 32^{1} \\
\mathrm{~F} 4^{1} 32^{1} \\
\mathrm{~F} 4{ }_{1}^{1} 32^{1} \\
\mathrm{I}^{1} 32^{1} \\
\mathrm{P} 4{ }^{1} 32^{1} \\
\mathrm{P}_{1}{ }_{1}^{1} 32^{1} \\
\mathrm{I}_{1}{ }_{1}{ }^{1} 32^{1}\end{array}$ & & & & & \\
\hline
\end{tabular}

\section{CONCLUSION}

Opechowki and Guccione gave 1191 types of symbols of magnetic space groups .D.B.LITIVN have tabulated 440 ferroelectric space groups by using Opechowki and Guccione symbols,also D.B.Litvin have tabulated 1,2 and 3 magnetic space groups. So here ferromagnetotoroidic space groups by using Opechowki and Guccione symbols are calculated. Ferromagnetotoroidic property is exhibited in solid nano crystal NaNbo3 with space group $\mathrm{p} 2{ }_{1} \mathrm{ma}\left(\mathrm{c}_{2 \mathrm{v}}\right)$

[1]. Aizu, K. (1970), Phys. Rev. B, @, 754-772

\section{REFERENCES}

[2]. Litvin, D.B., Acta Cryst. (2008) A 64, 316-320

[3]. Litvin, D.B., Acta, Cryst, (2007), Section A

[4]. Litvin, D.B.Acta. Cryst. (1968). A 42, 44-47

[5]. ASchER, E \& Janner, A.G.M... properties of Shubnikov point groups. Battelle institute, Geneva, Switzerlavel.

[6]. Rosa Lia Guccione, A Thesis of Magnetic Space groups

[7]. W. opechowski and R.Guccione, in magnetism, edited by G.T.Rado and H.Suhl (Academic, Newyork, 1965), Vol II A, P. 105.

[8]. Litvin. D.B.Ferroelectrics, 376: 158-167, 2008. 